\title{
Cáncer mamario y cervicouterino: el problema del absentismo femenino en la acción preventiva
}

\author{
Breast and cervical cancer: the problem of the lack of preventive actions in women \\ Carlos René Rodríguez-Garcés ${ }^{1 *}$ orcid.org/0000-0002-9346-0780 \\ Geraldo Bladimir Padilla-Fuentes ${ }^{1}$ orcid.org/0000-0003-0882-1818
}

1 Centro de Investigación CIDCIE, Universidad del Bío-Bío. Chillán, Chile

Fecha de recepción: Junio 06 - 2019

Fecha de revisión: Octubre 04 - 2019

Fecha de aceptación: Diciembre 19 - 2019

Rodríguez-Garcés CR, Padilla-Fuentes GB. Cáncer mamario y cervicouterino: el problema del absentismo femenino en la acción preventiva. Univ. Salud. 2020;22(1):41-51. DOI: https://doi.org/10.22267/rus.202201.173

\section{Resumen}

Introducción: La prevalencia del cáncer es un problema que vas más allá de la preocupación médica, por cuanto involucra aspectos subjetivos y sociales en la vida de quienes lo padecen o están en riesgo. Por esto, es de gran importancia detectarlo tempranamente, especialmente entre mujeres debido a su exposición a los factores de riesgo de cáncer de mama y cérvix. Objetivo: Analizar la abstención femenina en Chile a la Mamografía y Papanicolaou. Materiales y métodos: Se realizó un análisis multivariado de regresión logística binomial, de los datos disponibles en el Módulo de Salud de la Encuesta de Caracterización Socioeconómica Nacional (CASEN) en su edición del año 2015. Resultados: Se expone una generalizada abstención a la acción preventiva entre mujeres, aun cuando la prevalencia de ambos tipos de cáncer es alta. Los motivos personales aparecen como los más comunes para omitir Mamografía y/o Papanicolaou, especialmente la subvaloración de su importancia. Los factores que aumentan la probabilidad de abstención son la baja escolaridad, la ausencia de afiliación médica y la presencia de pareja. Conclusiones: Se plantea la necesidad de aumentar igualmente la cobertura del servicio preventivo, focalizar los recursos hacia la población vulnerable y mejorar las estrategias de incentivo hacia estos exámenes.

Palabras clave: Cáncer mamario; cáncer de cuello uterino; prevención; mamografía; prueba de papanicolaou. (Fuente: DeCS, Bireme).

\begin{abstract}
Introduction: The prevalence of cancer is a problem that goes beyond medical concern since it involves subjective and social aspects that affect the lives of those who either suffer from cancer or are at risk for it. Therefore, it is important to detect it early, especially among women due to their exposure to risk factors for breast and cervical cancer. Objective: To analyze women's abstention from mammography and Papanicolaou screening in Chile. Materials and methods: A multivariate binomial logistic regression analysis was conducted with available data from the Health Module of the National Socioeconomic Characterization Survey (NSCS) from 2015. Results: Even though there is a high prevalence of both types of cancer, there is a generalized female abstention from preventive actions. Personal reasons are the most common justifications given for avoidance of mammography and/or Papanicolaou test, especially undervaluing their importance. Factors that increase the probability of abstention include low education level, lack of medical affiliation, and presence of a partner. Conclusions: It is important to increase the coverage of preventive services, focus resources towards vulnerable populations, and create incentive strategies for women to take these exams.
\end{abstract}

Key words: Breast cancer; uterine cervical cancer; prevention; mammography; papanicolaou test. (Fuente: DeCS, Bireme).

\footnotetext{
*Autor de correspondencia

Carlos René Rodríguez-Garcés

e-mail: carlosro@ubiobio.cl
} 


\section{Introducción}

El cáncer de mama (CaMa) y cervicouterino $(\mathrm{CaCu})$ son las dos tipologías que más muertes causan entre mujeres. En la década de los 90' se estimaba que en América Latina y el Caribe el CaMa causaba alrededor de 300.000 muertes al año y actualmente es el tipo de cáncer más frecuente entre mujeres, seguido por el cáncer de pulmón y colon(1). Por su parte, el cáncer de cérvix es el tercero más común a nivel mundial, y en Sudamérica durante 2018 se diagnosticaron aproximadamente 56 mil $\operatorname{casos}^{(2)}$.

A pesar de los importantes avances tecnológicos y médicos, la prevalencia del cáncer entre la población se mantiente en niveles alarmantes( 3 ) es en torno a qué métodos de detección son mejores y, sobre todo en Europa y América, demanda hacia los gobiernos por medidas que protejan la salud, en especial para mujeres(4). Sustancialmente se solicita programas de salud pública que garanticen los medios para el diagnóstico temprano del cáncer, gratuitos y de calidad(5). Por lo demás, han incrementado los factores de riesgo vinculados a la aparición de estas patologías entre mujeres debido a nuevos estilos de vida, pautas alimentarias o consumo de hormonas como el estrógeno(6).

Los factores de riesgo, niveles de éxito y supervivencia en los tratamientos se encuentran asociados al nivel de desarrollo y producción económica de las regiones( 7,8$)$, lo que implica postergación para quienes habitan territorios empobrecidos y/o mal conectados con los centros urbanos(9). Gran parte de los casos diagnósticados y muertes por estos tipos de cáncer se producen en zonas con alta pobreza, precariedad en la atención médica, falta de instrumentos de diagnóstico y tamizaje; mortalidad que se ubica principalmente en África, Asia y América Latina y el Caribe(10-5).

La prevención, diagnóstico y tratamiento temprano del cáncer tiene una importancia fundamental para reducir su mortalidad y costos de tratamiento, mejorar la eficacia de la intervención médica, así como atenuar sus implicancias afectivas y psicológicas en la población(13,14). Pesquisas tardías impiden ubicar, interrumpir y/o extirpar carcinomas antes que causen algún daño irreversible en las mamas o el cuello uterino, lo que vuelve imprescindible asegurar la confiabilidad de estos métodos en beneficio de la sobrevida(15).
El éxito de las intervenciones preventivas de esta enfermedad reside, por una parte, en la selección, aplicación y ajuste de los instrumentos para su detección(16) y, por otra, en la iniciativa de las pacientes que hacen uso del tamizaje a través de servicios de seguridad social o campañas de promoción de salud.

En este sentido, la educación, sensibilización, concientización y vacunación, como es caso del Virus del Papiloma Humano(17), reducen la exposición a factores de riesgo en mayor medida que los métodos de prevención secundaria(18), como son la Mamografía y el Papanicolaou (PAP). Invertir recursos en la prevención del cáncer mitigaría su prevalencia y los altos costos de sus planes de cuidado(19), además de contribuir al autocuidado de la población en riesgo.

Por su bajo costo, aplicabilidad, sensibilidad y especificidad, tanto la Mamografía como el Papanicolaou son los métodos más utilizados para identificar anomalías en las mamas o en el cuello uterino(20,21), y han demostrado eficacia en la reducción de la mortalidad(22,23). Con las nuevas tecnologías, estos exámenes se actualizan y refinan, facilitando su inclusión a chequeos rutinarios y disminuyendo los eventuales inconvenientes derivados de su aplicación(24). Aunque no hay consenso respecto a la edad y frecuencia de los controles, en el caso de la Mamografía se recomienda sobre los 40 años(25), y para el Papanicolaou desde $\operatorname{los} 15$ años.

En Chile, según datos del Global Cancer Observatory para 2018, el CaMa registra tasas brutas de incidencia y mortalidad de 73,0 y 22,8 por cada 100.000 habitantes, mientras que para $\mathrm{CaCu}$ alcanzan el 21,0 y 9,8; cifras que al estar por debajo de las exhibidas por otros países ${ }^{(26,27)}$ generarían una menor percepción de riesgo entre las mujeres, desestimando la importancia del control preventivo. Ello, junto a inconvenientes de segmentación social y territorial, entraman dificultades para la eficacia de la atención preventiva, su calidad y cobertura según zona, comuna, género o edad(28).

En el caso de la Mamografía, exceptúando algunas carencias de cobertura e integración entre los centros de atención y un programa de respaldo efectivo(29), se encuentra que los motivos comunes para omitirla son desinformación (respecto de los 
exámenes y la enfermedad), la condición de afiliación a la seguridad social, el acceso y/o precariedad del servicio disponible, el temor al procedimiento y la duda sobre su importancia(30). Con el Papanicolaou la situación es similar, siendo la vulnerabilidad social, acceso restringido a los servicios de salud, situación laboral, baja escolaridad y ser joven, factores obstaculizadores ante la toma de decisión(31,32).

En este contexto, la acción preventiva y actitud de autocuidado cobra relevancia, más aún si se considera que desde 2009 el cáncer de mama es la primera causa de muerte oncológica entre mujeres chilenas(33). Someterse a exámenes preventivos es una acción fundamental para el hallazgo, diagnóstico y tratamiento del cáncer, no obstante las cifras actuales respecto de su uso están por debajo de lo esperado.

Dado que la vigilancia epidemiológica de estas enfermedades orienta la toma de desiciones para la política pública, este artículo tiene como objetivo analizar el comportamiento de abstención femenina a los exámenes de Mamografía y Papanicolaou en Chile, para lo cual se determinó cuáles son los motivos comunes para tal desición y qué factores aumentan su probabilidad de ocurrencia.

\section{Materiales y métodos}

\section{Diseño}

Se aplicó un diseño cuantitativo analítico transversal de carácter multivariante para las cohortes de la Encuesta de Caracterización Socioeconómica Nacional (CASEN) 2011 y 2015.

Se diseñaron y ajustaron sendos modelos de regresión logística binomial para analizar la situación de mujeres chilenas que no se han realizado los exámenes de Mamografía y Papanicolaou en los últimos 3 años.

\section{Población y muestra}

La unidad de observación refiere a mujeres en los rangos etarios recomendados para cada examen, es decir, mayores de 35 años en Mamografía para una población de referencia de 1.859.209 mujeres, y mayores de 15 años en Papanicolaou para una población de referencia de 2.705.964 mujeres. Dado su carácter muestral, se trabajó con segmentos representativos de la población para ambos conglomerados, los cuales fueron extraídos por muestreos estratificados y polietápicos a nivel nacional. En la exploración del Papanicolaou la muestra quedó establecida en 106.919 mujeres, mientras que en Mamografía fueron 70.412. El diseño muestral de CASEN 2015 acepta un error absoluto esperado de 0,5 puntos porcentuales y un error relativo esperado de $3,7 \%$ a nivel nacional (muestra objetivo total).

\section{Instrumento}

Se utilizaron los datos disponibles en la CASEN en su edición del año 2015, específicamente el Módulo de Salud, donde se pregunta acerca del padecimiento de enfermedades, las acciones preventivas correspondientes y los motivos de su abstención. Este instrumento de recolección de datos es una aproximación poblacional de la situación de los hogares nacionales a cargo del Ministerio de Desarrollo Social de Chile, aplicado bianualmente a una muestra representativa y de carácter nacional.

\section{Procedimiento}

Se analizaron índices acerca de la situación del cáncer en la población femenina, su prevalencia y distribución según rangos etarios reportados en CASEN 2015, comparando con datos correspondientes a la edición CASEN 2011, haciendo hincapié en los motivos que las mujeres reportan para no realizarse los exámenes preventivos. Estos fueron agrupados según las siguientes categorías:

-Información: hacen referencia al dominio y manejo de información clave para realizarse alguno de los exámenes preventivos.

-Personales: expresan la actitud, evaluación o barrera individual frente a los exámenes.

-Institucionales: situaciones vinculadas a la disponibilidad del servicio que impiden realizarlo.

El análisis se profundizó modelando el comportamiento de la abstención para ambos exámenes mediante regresión logística binomial, escogiéndose una serie de variables que, siendo teóricamente relevantes, están disponibles en CASEN. Los factores categoriales politómicos fueron transformados a variables dummy, fijando como categoría de referencia aquella que actúa como factor protector frente a la decisión de abstención. Para la estimación de la variable dependiente realización de examen preventivo, se incluyeron las 
siguientes variables independientes (Tabla 1) en el software estadístico SPSS (v.23).

Tabla 1. Variables incorporadas en los análisis

\begin{tabular}{|c|c|}
\hline Variable & Descripción \\
\hline Edad & $\begin{array}{l}\text { Variable ordinalizada en } 4 \text { categorías según rangos etarios teóricamente relevantes, siendo el rango “56 y más" la } \\
\text { categoría de referencia para variables dummy generadas. }\end{array}$ \\
\hline Nivel educativo & Variable ordinal de 4 categorías. "Educación superior" se usa de referencia para variables dummy. \\
\hline Pareja & Registra la "presencia" (0) o "ausencia" (Ref) de pareja con independencia de la naturaleza del vínculo. \\
\hline Zona & $\begin{array}{l}\text { Con base a la definición del Instituto Nacional de Estadística califica la residencia permanente sea "rural" o "urbana" } \\
\text { (Ref). }\end{array}$ \\
\hline Actividad & $\begin{array}{l}\text { Variable que explora la condición de participación en el mercado laboral expresada en las categorías "ocupada", } \\
\text { "desocupada", "inactiva" (Ref). }\end{array}$ \\
\hline NSE & $\begin{array}{l}\text { Nivel socioeconómico con base a los ingresos, salarios o rentas del hogar expresada en quintiles de ingresos siendo } \\
\text { el } 20 \% \text { más rico (“V quintil”) la categoría de referencia. }\end{array}$ \\
\hline PMD-5 & $\begin{array}{l}\text { Variable que expresa el estado de carencialidad del hogar dicotomizada con base a la métrica del Enfoque } \\
\text { Multidimensional de la Pobreza. Siendo "no pobre" la categoría de referencia. }\end{array}$ \\
\hline Afiliación & $\begin{array}{l}\text { Explora el tipo de plan de salud al que accede preferencialmente la mujer para objeto de su atención médica. } \\
\text { Variable dicotómica: "ninguna", "pública" y "privada" (Ref). }\end{array}$ \\
\hline
\end{tabular}

\section{Consideraciones éticas}

La investigación procesa información de fuentes secundarias, recogida por organismos oficiales de carácter ministerial, que resguardan la privacidad, confidencialidad de datos sensibles, por lo que esta investigación sería catalogada como "sin riesgo". Para el levantamiento y análisis de la información, se cumple con los protocolos definidos por la Comisión Nacional de Ciencia y Tecnología de Chile (CONICYT) y la Ley de Protección de datos de carácter personal (Ley $\mathrm{N}^{\circ}$ 19.628), los cuales se encuentran en sintonía con los lineamientos de declaraciones internacionales como la de Helsinki.

\section{Resultados}

La Tabla 2 reporta la información proporcionada por la Encuesta de Caracterización Socieconómica Nacional del año 2015 en su módulo de salud, se observa que 9 de cada 1000 mujeres chilenas declaran padecer o haber padecido algún tipo de cáncer, tasa que aumenta en prevalencia en rangos etarios mayores. Entre quienes padecen esta enfermedad, 1 de cada 2 mujeres tiene más de 55 años. Dentro de las tipologías Ca, los de mayor prevalencia son el de mama (CaMa) y cervicouterino $(\mathrm{CaCu})$, con un $54,8 \%$ y $28,6 \%$ respectivamente.

El CaMa concentra su presencia en la población femenina de mayor edad. El $57,8 \%$ de quienes presentan esta patología tienen más de 55 años. En este segmento etario afectado por algún $\mathrm{Ca}$, un $66,0 \%$ lo es de tipología mamaria, cifras significativamente mayores que las registradas en la población más joven.
En el caso del $\mathrm{CaCu}$, se evidencia un comportamiento epidemiológico contrario, cuyo perfil estaría más asociado a ciclos reproductivos, siendo el cáncer predominante en el grupo de mujeres que se encuentra entre los 26 y 34 años $(72,1 \%$ de los cánceres en mujeres).

Si bien se incluye sólo a partir del 2011 la pesquisa de este indicador por parte de la CASEN, las cifras informan que 1 de cada 2 mujeres mayores de 35 años no se había realizado una Mamografía en los últimos 3 años $(50,2 \%)$, y en el caso del Papanicolaou, para el segmento de mujeres mayores de 15 años, un 42,1\% no se lo había efectuado. En mediciones más recientes como la CASEN 2015, se informan similares déficits para Papanicolaou $(38,2 \%)$ y una disminución cercana a 10 puntos porcentuales para el caso de la Mamografía, situándose esta en el orden del 40,7\%.

En el grupo de mujeres entre los 15 y 25 años, alrededor del $69,3 \%$ de las mujeres no se realiza el examen; proporción que se revierte drásticamente al inspeccionar otras cohortes etarias. Las cifras manifiestan que en el rango de los 26 y 55 años es donde mayormente las mujeres ingresan al sistema de salud preventivo, coincidente con el ciclo de mayor actividad sexual y reproductiva.

Para el año 2015, independiente del grupo etario, en torno a 2 de cada 5 mujeres no se han efectuado la Mamografía en los últimos 3 años. Sin embargo, esto representa una reducción significativa en el déficit de cobertura respecto a 2011, que pasaron de un 
$50,2 \%$ a un $40,7 \%$ para 2015 , siendo especialmente notorio el impacto en la cohorte de 35-55 años.

Dada la importancia que revisten las razones de abstención para la planificación pública, la encuesta CASEN 2015 consulta a las mujeres las razones que motivaron su decisión de no realizarse Mamografía o Papanicolaou, como se aprecia en la Tabla 4.

Dentro de los motivos informados por mujeres, considerar o creer que no es necesario realizarse la Mamografía (30,9\%) o el Papanicolaou $(40,0 \%)$ es la principal razón de abstención, seguido por la falta de tiempo (CaMa=17,1\%; $\mathrm{CaCu}=16,4 \%)$ y el olvido $(\mathrm{CaMa}=16,5 \% ; \mathrm{CaCu}=16,7 \%)$. Estos tres motivos pertenecen a la dimensión Personal, la cual agrupa al
$87,4 \%$ de los casos que no se realiza Papanicolaou y al $81,6 \%$ que se abstiene a la Mamografía. Por su parte, el miedo o disgusto que provocaría realizarse estos exámenes es manifestado por alrededor del $12 \%$ de las mujeres que se abstiene, cuestión que pone el acento en otros motivos, pero siempre dentro de la dimensión Personal (Tabla 4).

Las razones de orden institucional y/o de falta de información, obstaculizadores clásicos y persistentes en las sociedades en vías de desarrollo, tienen en este estudio un peso marginal, bordeando el 15\% de los casos en ambos tipos de examen, no obstante, se observa en el factor institucional una prevalencia comparativa significativamente mayor en el caso del screening $\quad(11,1 \% \quad$ vs $\quad 5,2 \%)$.

Tabla 2. Estado del cáncer entre la población femenina chilena en 2015

\begin{tabular}{|c|c|c|c|c|c|c|c|c|}
\hline \multirow{2}{*}{ Edad } & \multicolumn{3}{|c|}{$\mathrm{CaCu}$} & \multicolumn{3}{|c|}{ CaMa } & \multicolumn{2}{|c|}{ Ca General } \\
\hline & Dist & Prev & Tasa* & Dist & Prev & Tasa* & Dist Gral. & Tasa* \\
\hline $15-25$ & 1,8 & 29,2 & 0,2 & 1,2 & 36,9 & 0,3 & 1,8 & 0,7 \\
\hline $26-34$ & 17,3 & 72,1 & 3,0 & 1,9 & 15,4 & 0,6 & 6,9 & 4,1 \\
\hline $35-55$ & 54,1 & 37,0 & 4,0 & 39,0 & 51,2 & 5,6 & 41,8 & 10,9 \\
\hline 56 y más & 26,8 & 16,0 & 2,3 & 57,8 & 66,0 & 9,6 & 48,1 & 14,6 \\
\hline Total & - & 28,6 & 2,6 & - & 54,8 & 4,9 & - & 8,9 \\
\hline
\end{tabular}

$\mathrm{Ca}=$ Cáncer; $\mathrm{Cu}=$ Cérvico uterino; Ma= Mama; Dist= Distribución; Prev= Prevalencia; Gral= General

Nota: Distribución= proporción de mujeres bajo condición Ca autoreportado expresado en porcentaje; Prevalencia= proporción de mujeres bajo condición Ca autoreportada respecto a total mujeres Ca en rango expresado en porcentaje; Tasa= proporción de mujeres bajo condición Ca autoreportado respecto a total mujeres en rango expresado en 1 de cada 1000.

Fuente: Encuesta de Caracterización Socioeconómica Nacional (CASEN) 2015.

Tabla 3. Déficit de cobertura preventiva según grupo etario (Porcentaje)

\begin{tabular}{|c|c|c|c|c|c|c|c|c|}
\hline \multirow{3}{*}{ Edad } & \multicolumn{4}{|c|}{ Papanicolaou } & \multicolumn{4}{|c|}{ Mamografía } \\
\hline & \multicolumn{2}{|c|}{2011} & \multicolumn{2}{|c|}{2015} & \multicolumn{2}{|c|}{2011} & \multicolumn{2}{|c|}{2015} \\
\hline & Dist & Prev & Dist & Prev & Dist & Prev & Dist & Prev \\
\hline $15-25$ & 39,8 & 71,8 & 37,7 & 69,3 & - & - & - & - \\
\hline $26-34$ & 8,2 & 24,3 & 8,7 & 22,7 & - & - & - & - \\
\hline $35-55$ & 19,5 & 23,2 & 17,9 & 19,7 & 53,4 & 46,8 & 49,9 & 37,9 \\
\hline 56 y más & 32,5 & 50,5 & 35,7 & 45,7 & 46,6 & 53,4 & 50,1 & 43,9 \\
\hline Total & - & 42,1 & - & 38,2 & - & 50,2 & - & 40,7 \\
\hline
\end{tabular}

Dist= Distribución; Prev= Prevalencia

Nota: Distribución= proporción de mujeres bajo déficit preventivo dentro de tipo de examen expresado en porcentaje; Prevalencia= proporción de mujeres bajo déficit preventivo dentro de categoría expresado en porcentaje.

Fuente: Encuesta de Caracterización Socioeconómica Nacional (CASEN) 2015

Tabla 4. Motivos por los cuales las mujeres no se realizan examen preventivo (Porcentaje)

\begin{tabular}{lrr}
\hline \multicolumn{1}{c}{ Motivos según tipología } & Mamografía & Papanicolaou \\
\hline Información & 1,2 \\
No sabe dónde hacérsela & 5,5 \\
No sabía que tenía que hacerse ese examen & 0,7 \\
No conoce ese examen & 5,9 \\
Personales & 1,2 \\
Le da miedo o le disgusta & 12,6 \\
Se le olvida hacérselo & 16,5 \\
No cree que lo necesite & 30,9 \\
No tiene tiempo & 17,1 \\
No tiene dinero & 4,5 \\
Institucional & 12,5 \\
El horario del consultorio no le sirve & 16,7 \\
No ha podido conseguir hora & 16,4 \\
\hline
\end{tabular}

Fuente: Encuesta Caracterización Socioeconómica Nacional (CASEN) 2015. 
Con la finalidad de abordar los factores que pueden influir en la abstención, y considerando la complejidad multidimensional que subyace en panoramas decisionales de esta naturaleza, se elaboraron modelos estadísticos de regresión logística binomial incorporando las variables teóricamente relevantes (Tabla 5).

En razón de los resultados, son elementos importantes para las mujeres al momento de decidir si someterse o no a algún examen, variables como la edad, el nivel educativo, la tenencia de pareja, zona de residencia, condición laboral, afiliación de salud y situación socioeconómica, definida esta última desde la perspectiva de ingresos económicos (Quintil de ingresos) y enfoque multidimensional (PMD-5).

En lo que a Papanicolaou refiere, todos los rangos de edad reportan coeficientes de regresión estadísticamente significativos $(p<0,01)$, aunque con comportamientos diferenciados. Así, mientras en los grupos de menor y mayor edad el riesgo de abstención aumenta, en los rangos intermedios disminuye; resultado que es consiste con el observado en el contexto bivariado en su análisis de porcentaje.

En la Mamografía, se observa una relativa mayor probabilidad de mujeres de tercera edad de someterse al examen respecto de aquellas más jóvenes. La edad, aunque significativa estadísticamente $(p<0,01)$, presenta una magnitud conservadora $[\operatorname{EXP}(\beta)=1,014]$.

En los modelos planteados, a medida que aumenta el nivel educativo de la mujer disminuye la probabilidad de manifestar riesgo de abstención en ambos exámenes $(p<0,01)$, situación más acentuada en el caso del Papanicolaou. Así también, la ausencia de pareja se constituye en un factor de riesgo. En efecto, mujeres que viven solas son más proclives a marginarse del control preventivo del CaMa, donde cerca de 1 de cada 2 mujeres en esta condición se abstiene $(47,4 \%)$, representando en el modelo una probabilidad de un $59 \%$ superior a la de mujeres emparejadas.

Aunque con una incidencia menos acentuada a la observada en otros factores, curiosamente el vivir en el ámbito urbano se traduce en un factor de riesgo $(p<0,01)$. Igualmente, frente al supuesto de que la inserción laboral podría marginar a las mujeres del control preventivo en razón del déficit horario, se encontró que en comparación a quienes se han insertado al menos una vez en el mercado laboral, son las mujeres inactivas las más propensas a la abstención en ambos exámenes.

Tabla 5. Modelo de regresión logística para factores que inciden sobre la abstención a Mamografía y Papanicolaou

\begin{tabular}{|c|c|c|c|c|}
\hline \multirow[b]{2}{*}{ Factores } & \multicolumn{2}{|c|}{ Papanicolaou } & \multicolumn{2}{|c|}{ Mamografía } \\
\hline & $\begin{array}{c}\% \\
\text { Abs. }\end{array}$ & $\operatorname{Exp}(\beta)$ & $\begin{array}{c}\% \\
\text { Abs. }\end{array}$ & $\operatorname{Exp}(\beta)$ \\
\hline \multicolumn{5}{|l|}{ Edad } \\
\hline $15-25$ & 69,3 & $2,622^{* *}$ & - & - \\
\hline $26-35$ & 22,7 & $0,502^{* *}$ & - & - \\
\hline $36-55$ & 19,7 & $0,419^{* *}$ & 37,9 & $1,014^{* *}$ \\
\hline 56 y más & 45,7 & Ref & 43,9 & Ref \\
\hline \multicolumn{5}{|l|}{ Nivel educativo } \\
\hline Sin Ed. formal & 63,0 & $2,637^{* *}$ & 62,6 & $1,975^{* *}$ \\
\hline Hasta básica & 39,0 & $1,395^{* *}$ & 46,2 & $1,186^{* *}$ \\
\hline Hasta media & 38,8 & $1,239^{* *}$ & 39,0 & $1,024 * *$ \\
\hline Superior o más & 34,5 & Ref & 31,2 & Ref \\
\hline \multicolumn{5}{|l|}{ Pareja } \\
\hline $\mathrm{Si}$ & 25,1 & $0,471^{* *}$ & 35,3 & $0,630^{* *}$ \\
\hline No & 50,0 & Ref & 47,4 & Ref \\
\hline \multicolumn{5}{|l|}{ Zona } \\
\hline Rural & 37,3 & $0,904^{* *}$ & 43,0 & $0,915^{* *}$ \\
\hline Urbana & 38,4 & Ref & 40,3 & Ref \\
\hline \multicolumn{5}{|l|}{ Actividad } \\
\hline Ocupada & 26,7 & $0,598^{* *}$ & 35,7 & $0,759 * *$ \\
\hline Desocupada & 39,7 & $0,734^{* *}$ & 41,6 & $0,827^{* *}$ \\
\hline Inactiva & 47,6 & Ref & 44,7 & Ref \\
\hline \multicolumn{5}{|l|}{ NSE } \\
\hline I & 41,6 & $0,943^{* *}$ & 48,9 & $1,573^{* *}$ \\
\hline II & 40,5 & $0,990^{* *}$ & 44,7 & $1,454^{* *}$ \\
\hline III & 39,7 & $1,065^{* *}$ & 41,3 & $1,329 * *$ \\
\hline IV & 36,8 & $1,099^{* *}$ & 37,6 & $1,290^{* *}$ \\
\hline $\mathrm{V}$ & 30,5 & Ref & 28,1 & Ref \\
\hline \multicolumn{5}{|l|}{ PMD-5 } \\
\hline No pobre & 37,6 & Ref & 39,3 & Ref \\
\hline Pobre & 41,0 & $1,010^{* *}$ & 46,7 & $1,140^{* *}$ \\
\hline \multicolumn{5}{|l|}{ Afiliación } \\
\hline Privada & 31,7 & Ref & 25,3 & Ref \\
\hline Pública & 39,0 & $1,167^{* *}$ & 43,1 & $1,578^{* *}$ \\
\hline Ninguna & 48,7 & $1,782^{* *}$ & 51,6 & $2,344^{* *}$ \\
\hline \multicolumn{5}{|c|}{ Resumen del modelo } \\
\hline $\mathrm{R}^{2}$ de Cox y Snell & & 0,183 & & 0,047 \\
\hline $\mathrm{R}^{2}$ de Nagelkerke & & 0,249 & & 0,064 \\
\hline
\end{tabular}

Nota: Ed= Educación; NSE = Nivel Socioeconómico; PMD-5= Pobreza Multidimensional según metodología Alkire y Foster en 5 dimensiones; \% Abs. = Porcentajes de Abstención que representa la proporción de mujeres dentro de la categoría del regresor que declara no realizarse el examen. Ref= categoría de referencia en la construcción de variables dummy para el análisis de regresión logística binomial. Sensibilidad= proporción de mujeres que no se realizan el examen correctamente identificadas; Especificidad= proporción de mujeres que si se realizan el examen y están correctamente clasificadas.

**= significativo al 0,01

Fuente: Encuesta de Caracterización Socioeconómica Nacional (CASEN) 2015. 


\section{Discusión}

En consistencia con estos datos, estudios epidemiológicos dan cuenta de la mayor prevalencia de CaMa entre mujeres adultas, junto a un aumento en tasa de mortalidad los últimos 25 años; evento que estaría asociado a una mayor exposición a determinados factores de riesgo y transiciones en las pirámides demográficas.

En el caso del $\mathrm{CaCu}$, se evidencia un comportamiento epidemiológico contrario, cuyo perfil estaría más asociado a ciclos reproductivos, siendo el tipo de cáncer predominante en el grupo de mujeres jóvenes ( 26 y 34 años). El cáncer de cérvix está asociado con la presencia del VPH, agente patógeno de transmisión sexual cuyo riesgo de exposición se acrecienta conforme aumenta el número de parejas sexuales y en mujeres en edad fértil.

Por otra parte, los factores de riesgo y el perfil epidemiológico del cáncer de mama, presentando etiológicamente un componente de raíz genética, están asociados a mujeres de mayor edad y la ausencia de amamantamiento en condición de alta paridad(34). Exposición a factores de riesgo que determinan que en Chile mueran anualmente alrededor de 1000 mujeres por CaMa, representando la segunda causa de muerte por enfermedades malignas en la mujer.

La prevalencia de cáncer descrita entre población femenina y su paulatino aumento de mortalidad podrían ser reducidos significativamente a través de acciones preventivas, tal como son la vacunación contra el VPH en preadolescentes y los programas de tamizaje. En Chile existe un abanico de programas públicos de prevención al cáncer de acceso gratuito, especialmente focalizados a población en riesgo(23). Medidas de política pública que, contrario a lo esperado, exhiben déficit en sus niveles de cobertura entre grupos de riesgo, tal como indica la Tabla 3.

Si bien Mamografía y Papanicolaou son ambulatorios, escasamente invasivos y de prestación gratuita, la responsabilidad en el autocuidado varía en razón de múltiples factores. En el caso del PAP, la falta de información, la iniciación sexual tardía, la ausencia de vinculación entre establecimientos educativos y centros de salud, y la escasa educación sexual, pueden llevar a las mujeres jóvenes a presentar bajos niveles de percepción de riesgo(35).
Como el cáncer de mama se asocia con la entrada a la adultez tardía, su control comienza a los 35 años, lo cual podría contribuir a que mujeres en esta etapa se sometan al examen en razón de la mayor información manejada respecto a su riesgo. Es probable que el acceso al servicio, el temor al procedimiento y/o los resultados sean relevantes al momento de decidir si realizarse o no el examen. Al respecto, la ejecución de programas de salud pública focalizados en las barreras de información y costos que inhiben la acción preventiva pudieron haber posibilitado la reducción significativa en los déficit de cobertura(22).

La cobertura de la acción preventiva difiere en razón del tipo de cáncer, la etapa de ciclo vital y el año al que haga referencia la medición. La materialización de la acción preventiva es, en última instancia, una decisión de carácter personal, motivada por un conjunto de factores de tipología y envergadura muy variada. En esta elección conviven elementos estructurales de carácter institucional con sistemas de creencia y/o perfiles actitudinales. En efecto, a las tradicionales barreras de acceso, distancia de los centros de atención y dificultades para ser atendido oportunamente, se suman factores de orden cultural, creencias populares y baja percepción del riesgo(36).

La alta prevalencia que adquieren los motivos de orden personal serían expresión de una baja percepción de riesgo hacia la ocurrencia del cáncer, lo que resta importancia a la acción preventiva, más aún si se considera que el desarrollo cultural ha propiciado la desarticulación de barreras de resistencia por pudor o vergüenza a estos exámenes en edades tempranas(5).

La menor relevancia que suscitan las categorías de restricción institucional o deficiente manejo de información develarían que, en lo que a diagnóstico y tratamiento del cáncer refiere, el déficit de cobertura estaría más asociado a un factor de autorresponsabilidad y predisposición de la mujer que a la sofisticación o promoción de los mecanismos institucionales de detección precoz. Similares inferencias han sido realizadas por Ponce(17), Serra ${ }^{(29)}$ y Wiesner(37), entre otros.

Los homogéneos valores en la tipología informativa, darían cuenta de una base cultural de conocimientos relativamente compartidos respecto de la acción preventiva, pese a los diferenciales perfiles etarios 
de la población objetivo a los que se circunscriben estos exámenes.

La abstención, en consecuencia, sería resultado de una acción racional en la que intervienen factores cognitivos, actitudinales y de perfil conductual, decisión que subvalora las eventuales implicancias de riesgo patológico. A menudo, los comportamientos de riesgo son resultado de creencias erróneas o inexactas sobre la enfermedad y/o el examen, acentuadas por el déficit de información desde las instituciones encargadas de la promoción y orientación del autocuidado(38).

Si bien actitudes positivas hacia el examen no implican necesariamente la manifestación de la conducta, si logran predecir una mayor probabilidad de realizarlo. Una predisposición favorable puede no ser suficiente, pero si necesaria para que las mujeres se tomen el examen y lo repitan. De allí la importancia que reviste para el autocuidado la promoción que los organismos de salud y sus profesionales realicen en torno a la sensibilización de su población objetivo(22).

Por otra parte, el comportamiento cóncavo de la edad en el análisis de abstención a PAP se vincularía con las principales causas del $\mathrm{CaCu}$ y el cambio en los ciclos reproductivos, ya que mujeres de tercera edad están expuestas a menor riesgo de padecer esta enfermedad que las jóvenes; en consiguiente, optarían por marginarse del procedimiento. Para aquellas en el umbral de los 25 años, la abstención puede estar vinculada al desinterés o la asociación de la enfermedad con etapas más tardías del ciclo vital. Tanto la orientación médica como la difusión de los síntomas de cáncer en los medios de comunicación de masas pueden llevar a las mujeres en etapas vitales tardías a preocuparse por tomar el examen, especialmente si conjuga con experiencias cercanas de padecimiento de la enfermedad $o$ exposición a exámenes preventivos similares(39).

La educación en términos aditivos se configura en un factor de protección. El desarrollo educativo apareja una mayor comprensión por parte de las personas respecto de las prácticas básicas de salud y un mayor riesgo percibido de la amenaza potencial que supone el cáncer, lo que articularía, en mayor proporción respecto de aquellas mujeres con baja escolaridad, conductas preventivas. Por lo demás, el capital social derivado de un mayor nivel educativo densifica las redes de exposición, ya sea por experiencias ligadas al cáncer o a la realización del examen, facilitando así la toma de esta decisión(35).

$\mathrm{Al}$ estar el $\mathrm{CaCu}$ vinculado principalmente con hábitos sexuales, y dada la educación sexual que se ha incorporado paulatinamente en los programas educativos(7), es esperable su incidencia en la manifestación de la conducta preventiva. El hecho de no establecer una relación sentimental aumenta el riesgo de abstención. Comprensible en el caso del PAP por cuanto mujeres no expuestas a actividad sexual frecuente, pueden desestimar la importancia del examen. Igual tendencia inhibidora, aunque menos acentuada, se observa respecto de la Mamografía.

Los perfiles etarios de la población objetivo son distintos según tipología de examen, registrándose para el caso de la Mamografía un rango de edad más acotado y una mayor tendencia a encontrar adultas mayores que viven solas. En comparación a quienes habitan sectores rurales, las mujeres urbanas tienen más probabilidades de no someterse a alguno de los exámenes preventivos. Hallazgo contradictorio toda vez que acceso, tecnología, organización y atención oportuna en materias de salud se asocian a la urbe y a su densidad poblacional. La disposición del servicio, así como la oportunidad en la atención, está en relación con la infraestructura disponible, presupuesto y capacidad de atención, atributos de mayor prevalencia en centros urbanos. Si bien las inferencias a este respecto hay que tomarlas con cautela, la escasa diferencia porcentual observada entre mujeres rurales y urbanas estaría informando de una mayor cobertura y equidad en el acceso a la acción preventiva. La existencia de una población beneficiaria más acotada y las acciones de derivación por interconsulta a centros de mayor complejidad, podrían dar luces para comprender el comportamiento de las mujeres rurales a este respecto, toda vez que la literatura informa que es precisamente en estos sectores donde el acceso al sistema sanitario es más limitado por distancia, necesidad de transporte o tiempo, además de existir una menor disponibilidad de acceso a programas educativos sobre el cáncer(40).

El mayor riesgo de abstención a PAP en mujeres inactivas puede estar vinculado al comportamiento observado en el nivel de estudios, por cuanto una baja escolaridad apareja menores posibilidades de 
empleo y afecta las condiciones de vida, acceso y atención médica. Aun cuando la falta de recursos generados por la mujer inactiva podría condicionar su acceso al servicio, ambos exámenes tienen garantía de gratuidad, por lo cual podríamos apoyar la tesis que una mayor inserción social de la mujer mediante alta escolaridad y actividades laborales apareja un mayor manejo de hábitos básicos de salud preventiva, distinto de quienes circunscriben a los espacios domésticos(6).

La cesantía y falta de ingresos generan discontinuidad de la adscripción a los sistemas de salud previsional, quedando al margen de atenciones médicas adicionales al plan básico de salud general. Bajo esta idea, se encuentra que la afiliación previsional es un factor protector ante la acción preventiva, especialmente en el sector privado. Dada la segmentación del sistema de salud previsional, los centros privados tienden a contar con una mejor infraestructura y equipamiento, siendo percibidos como más eficientes que el sector público en la tarea de acerca a las mujeres a la acción preventiva, a quien se le califica como burocrático y lento(30).

Si bien ambas pruebas cuentan con garantías de gratuidad, igualmente la disponibilidad de recursos económicos es un factor protector ante la realización de la Mamografía, distinto al Papanicolaou, donde muestra un comportamiento conservador. La carencia de ingresos, presumiblemente asociada a la vulnerabilidad y en las condiciones sociales actuales, se caracteriza por limitar el acceso de las personas a determinados servicios sociales. En el caso de la Mamografía, que requiere instrumentos e instalaciones más refinadas para su cumplimiento que el Papanicolaou, tal marginación puede ser extensión de un aislamiento generalizado del sistema de salud, más aún si se trata de uso de tecnologías y procedimientos más allá del pabellón común(39).

Al expandir la perspectiva de la pobreza desde la insuficiencia de ingresos familiares hacia la carencia del hogar en determinados servicios, se encuentra que mujeres ubicadas en grupos familiares vulnerables tienen mayor probabilidad de aislarse que aquellas no carentes. Esta variable, que representa la falta en el hogar de acceso a servicios de salud, vivienda, educación, trabajo y previsión social, es correlato de lo anteriormente expuesto y resume el impacto que tiene el status social sobre las posibilidades de prevención sanitaria(30).

\section{Conclusiones}

El control preventivo del cáncer es un problema que trasciende el ámbito médico y se instala en el centro de la vida social como enfermedad catastrófica. En paralelo a la discusión acerca de la seguridad y eficacia de los mecanismos de detección temprana del cáncer, la abstención hacia la realización de la Mamografía o el Papanicolaou es una situación preocupante que se vincula con el perfil social y actitudinal de las mujeres, combinando variables demográficas y características estructurales, con ideas y creencias asociadas a la acción preventiva.

Como se expuso, dentro de los tipos de cáncer presentes en la población femenina son el $\mathrm{CaCu}$ y el CaMa los más frecuentes, especialmente en los grupos mayores de 35 años.

Esta alta prevalencia en $\mathrm{CaCu}$ y el CaMa no se condicen con los todavía bajos niveles de adhesión que tiene la acción preventiva, no obstante, las tasas de cobertura han aumentado significativamente respecto del año 2011.

Al analizar los motivos esgrimidos por las mujeres que han decidido no someterse a alguno de los exámenes analizados, la categoría de "motivos personales" es la más significativa. En lo inmediato, esto tiene dos implicancias; primero, que se han reducido las limitantes informativas e institucionales que otrora ocupaban un lugar importante $y$ obstaculizaban tal decisión; segundo, que la instalación de una cultura preventiva es tarea pendiente de la política pública, la cual debe apuntar hacia la modificación de ideas y creencias no suficientemente fundadas, así como a la educación respecto de conductas de riesgo en las mujeres.

Consistente con lo anterior, el análisis multivariante informa que son factores de riesgo frente a la abstención el tener un bajo nivel de escolaridad, vivir en situación de pobreza, no tener pareja, así como el no participar del mercado laboral, ni tener afiliación a un sistema de salud determinado.

En síntesis y a la luz de los datos, el accionar de la política pública en esta materia, junto con subsanar 
las eventuales restricciones institucionales con una mayor dotación de equipos y prolongación de la gratuidad, debiera desarrollar programas de intervención socioeducativas que produzcan un cambio en los perfiles actitudinales de mujeres que manifiestan conductas de riesgo frente a la acción preventiva, intervención que debiese estar focalizada preferencialmente hacia mujeres que registran uno o más atributos catalogados como no protectores en este estudio.

\section{Referencias}

1. Siegel R, Miller K, Jemal A. Cancer Statistics, 2018. CA Cancer J Clin. 2018;68(1), 7-30. DOI: 10.3322/caac.21442.

2. Bray F, Ferlay J, Soerjomataram I, Siegel RL, Torre LA, Jemal A. Global cancer statistics 2018: GLOBOCAN estimates of incidence and mortality worldwide for 36 cancers in 185 countries. ACS Journals. 2018;64(6):394-424. DOI: 10.3322/caac.21492

3. Bray F, Colombet M, Mery L, Piñeros M, Znaor A, Zanetti R and Ferlay J. Cancer Incidence in Five Continents. XI (electronic version). Lyon: International Agency for Research on Cancer. 2017. Disponible en: http://ci5.iarc.fr/CI5-XI/Default.aspx

4. Yahyazadeh S, Mehraeen R. A comparison of the diagnostic value of magnetic resonance mammography versus ultrasound mammography in moderate and high-risk breast cancer patients. Journal of Evolution of Medical and Dental Sciences, 2018;53, 5629-5633.

5. Torres G, Ortega C, Llerenas A, Villalobos A, Salmeron J, Lazcano E, Hernández M. Patrones de utilización de programas de prevención y diagnóstico temprano de cáncer en la mujer. Revista de Salud Pública, México. 2013; 55. Disponible

en: http://www.scielo.org.mx/scielo.php?script=sci_arttext\&pi $\mathrm{d}=$ S0036-36342013000800022

6. Icaza G, Núñez L, Bugueño H. Descripción epidemiológica de la mortalidad por cáncer de mama en mujeres en Chile. Revista Médica de Chile. 2017;145(1), 106-114. doi.org/10.4067/S0034-98872017000100014

7. Organización Mundial de la Salud (OMS). Mejor detección, tratamiento y vacunas asequibles para prevenir muertes por cáncer cervicouterino. 2009. Disponible en: https://www.paho.org/hq/index.php?option=com_content \&view=article\&id=7815:2008-2009-better-screeningaffordable-can-prevent-cancer-

deaths\&Itemid $=4327$ \&lang $=\mathrm{es}$

8. DeSantis CE, Lin CC, Mariotto AB, Siegel RL, Stein KD, Kramer JL, Alteri R, Robbins AS, Jemal A. Cancer treatment and survivorship statistics, 2014. Cancer J Clin. 2014; 64(4), 252-271. DOI: 10.3322/caac.21235

9. Gonzaga C, Freitas R, Souza M, Curado M, Freitas N. Disparities in female breast cancer mortality rates between urban centers and rural areas of Brazil: ecological timeseries study. Breast. 2014;23, 180-187. DOI: 10.1016/j.breast.2014.01.006

10. Ferlay J, Shin HR, Bray F, Forman D, Mathers C, Parkin DM. Estimates of worldwide burden of cancer in 2008: GLOBOCAN 2008. International Journal of Cancer. 2010;127(12), 2893-2917. DOI: 10.1002/ijc.25516
11. Leach CR, Weaver KE, Aziz NM, Alfano CM, Bellizzi KM, Kent EE, Forsythe LP, Rowland JH. The complex health profile of long-term cancer survivors: prevalence and predictors of comorbid conditions. Journal of Cancer Survivorship. 2015; 9(2), 239-251. DOI: 10.1007/s11764-014-0403-1

12. Renna N, De Azevedo G. Late-Stage Diagnosis of Breast Cancer in Brazil: Analysis of Data from Hospital-Based Cancer Registries. Revista Brasileira de Ginecologia e Obstetrícia/RBGO Gynecology and Obstetrics. 2018;40(3), 127-136. DOI: 10.1055/s-0038-1624580

13. Koch L, Bertram H, Eberle A, Holleczek B, Schmid-Höpfner S, Waldmann A, Arndt V. Fear of recurrence in long-term breast cancer survivors-still an issue. Results on prevalence, determinants, and the association with quality of life and depression from the Cancer Survivorship-a multi-regional population-based study. Psychooncology, 2014;23(5), 547-554. DOI: 10.1002/pon.3452

14. Simard S, Savard J. Screening and comorbidity of clinical levels of fear of cancer recurrence. Journal of Cancer Survivorship. 2015;9(3):481-491. DOI: $10.1007 /$ s11764015-0424-4

15. Siu A. Screening for breast cancer: US Preventive Services Task Force recommendation statement. Annals of Internal Medicine. 2016;164(4), 279-296. DOI: 10.7326/M15-2886

16. Ma X, Zhang J, Zhong W, Shu C, Wang F, Wen J, Zhou M, Sang Y, Jiang Y, Liu L. The diagnostic role of a short screening tool-the distress thermometer: a meta-analysis. Supportive Care in Cancer. 2014; 22(7), 1741-1755. DOI: 10.1007/s00520-014-2143-1

17. Ponce M. La prevención del cáncer de cuello de útero y de mama en servicios de salud y organizaciones no gubernamentales de la ciudad Autónoma de Buenos Aires. Salud Colectiva. 2013; 9(2), 215-233. Disponible en: https://www.redalyc.org/pdf/731/73129415007.pdf

18. Fica A. Prevención del cáncer cérvico-uterino en Chile: mucha vacuna y poco Papanicolau. Revista Chilena de Infectología, 2014;31(2), 196-203. doi.org/10.4067/S071610182014000200010

19. Boekhout A, Maunsell E, Pond G, Julian J, Coyle D, Levine M, Grunfeld E. A survivorship care plan for breast cancer survivors: extended results of a randomized clinical trial. Journal of Cancer Survivorship. 2015; 9(4), 683-691. DOI: 10.1007/s11764-015-0443-1

20. Ramírez T, López A, Arancibia P, Sáez C, Díaz L, Taub T, Moyano L. Mastitis granulomatosa idiopática: 10 años de experiencia en el Centro de Imagenología del Hospital Clínico de la Universidad de Chile. Revista Chilena de Obstetricia y Ginecología. 2015; 80(2), 111-118. http://dx.doi.org/10.4067/S0717-75262015000200003

21. Le M, Mothersill C, Seymour C, McNeill F. Is the falsepositive rate in mammography in North America too high? The British journal of radiology. 2016;89. DOI: 10.1259/bjr.20160045

22. Chile, Ministerio de Salud. Guía Clínica AUGE: Examen medicina preventiva. Santiago de Chile. 2013. Disponible en: https://www.minsal.cl/examen-medicina-preventiva/

23. Uchida M. Mamografía de screening y realidad chilena. Revista Chilena de Radiología. 2008; 14(3), 130-134. http://dx.doi.org/10.4067/S0717-93082008000300005

24. Bellolio E, Pineda V, Burgos M, Iriarte MJ, Becker R, Araya J, Villaseca M, Mardones N. Valor predictivo del sistema BIRADS para detección de cáncer de mama con Mamografía en biopsias por punción con aguja gruesa: Revisión de 5 
años en un hospital de referencia. Revista Médica de Chile. 2015; 143(12), 1533-1538. http://dx.doi.org/10.4067/S0034-98872015001200005

25. Jørgensen $K$, Kalager $M$, Barratt A, Baines C, Zahl P, Brodersen J, Harris R. Overview of guidelines on breast screening: Why recommendations differ and what to do about it. The Breast. 2017; 31, 261-269. DOI: 10.1016/j.breast.2016.08.002

26. Ferlay J, Soerjomataram I, Dikshit R, Eser S, Mathers C, Rebelo M, Parkin DM, Forman D, Bray F. Cancer incidence and mortality worldwide: sources, methods and major patterns in GLOBOCAN 2012. International Journal of Cancer, 2015; 136(5), 359-86. DOI: 10.1002/ijc.29210

27. Organización para la Cooperación y el Desarrollo Económico (OCDE). Health at a Glance 2013: OECD Indicators. Paris: OECD Publishing.

28. Núñez L, Icaza G. Calidad de las estadísticas de mortalidad en Chile, 1997-2003. Revista Médica de Chile. 2006; 134, 1191-1196. 98872006000900016

29. Serra I. Cáncer de mama en Chile: pasado, presente y futuro. Revista Chilena de Cirugía. 2011; 63(6), 553-556. http://dx.doi.org/10.4067/S0718-40262011000600001

30. Charry L, Carrasquilla G, Roca S. Equidad en la detección del cáncer de seno en Colombia. Revista Salud Pública. 2008;10(4), 571-582. Disponible en: http://www.scielo.org.co/scielo.php?script=sci_abstract\&pi $\mathrm{d}=$ S0124-00642008000400007

31. Arzuaga MA, Souza MdL, Azevedo V. El cáncer de cuello de útero: un problema social mundial. Revista Cubana de Enfermería. 2012; 28(1), 63-73. Disponible en: http://scielo.sld.cu/scielo.php?script=sci_arttext\&pid=S086 4-03192012000100007

32. Sankaranarayanan R, Esmy P, Rajkumar R, Muwonge R, Swaminathan R, Shanthakumari S, Cherian J. Effect of visual screening on cervical cancer incidence and mortality in Tamil Nadu, India: a cluster-randomised trial. The Lancet. 2007; 370, 398-406. DOI: 10.1016/S0140-6736(07)61195-7
33. Navarro M, Razmilic D, Araos I, Rodrigo A, Andia M. Rendimiento de la Mamografía espectral de energía dual con contraste en la detección de cáncer de mama: experiencia en un centro de referencia. Revista Médica de Chile. 2018; 146, 141-149. 98872018000200141

34. Peña Y, González M, Avila D, Utria L, Más Y. Factores de riesgo para padecer cáncer de mama en la población femenina. Revista Finlay. 2017; 7(4). http://scielo.sld.cu/scielo.php?script=sci_abstract\&pid=S22 21-24342017000400008\&lng=es\&nrm=iso

35. Rubio D. Proceso de adopción de la conducta saludable de toma de citología vaginal en mujeres jóvenes. Rev. salud. bosque. $\quad 2017 ; \quad 7(1), \quad 9-17$. https://doi.org/10.18270/rsb.v7i1.2090

36. Picón M. Conocimientos, percepciones, y prácticas de mujeres jóvenes acerca del papiloma virus humano. Independent Study Project (ISP) Collection. 2009; 732, 5-33. Disponible https://digitalcollections.sit.edu/isp_collection/732

37. Wiesner C. Determinantes psicológicos, clínicos y sociales del diagnóstico temprano del cáncer de mama en Bogotá, Colombia. Revista Colombiana de Cancerología. 2007;11(1), 13-22. Disponible en: http://www.imbiomed.com/1/1/articulos.php?method=sh owDetail\&id_articulo=49342\&id_seccion=1161\&id_ejempla $\mathrm{r}=4999$ \&id_revista $=92$

38. Montoya A, Macías N. Creencias irracionales y su relación con la autoestima en pacientes con diagnóstico de cáncer mamario. Ambato, Ecuador: Escuela de Psicología. Pontificia Universidad Católica del Ecuador. 2015. Disponible en: http://repositorio.pucesa.edu.ec/handle/123456789/1255

39. Urrutia M. Creencias sobre Papanicolaou y cáncer cérvicouterino en un grupo de mujeres chilenas. Revista chilena de obstetricia y ginecología. 2012; 77(1), 3-10. http://dx.doi.org/10.4067/S0717-75262012000100002

40. López A. La ruralidad como determinante de la demora diagnóstica en el cáncer de mama. Cad Aten Primaria. 2009; 16(3), 193-196. Disponible en: https://www.researchgate.net/publication/43105907_La_r uralidad_como_determinante_de_la_demora_diagnostica_en_ el_cancer_de_mama 